\title{
Membres de la famille coloniale
}

En arrivant aux Antilles, Du Tertre et les autres missionnaires s'estiment « heureux de souffrir quelque chose pour la gloire de Jésus-Christ en secourant ses membres ». (1667, tome I : 155) L'un endure les maladies et la faim tandis que l'autre souffre en aidant ses frères, et ces formes de supplices contribuent toutes deux à révéler la présence sublime de Jésus-Christ dans le Nouveau Monde et à constituer la communauté coloniale. La notion de membre est ici à comprendre dans son double sens physique et communautaire. Dans son livre Les Renaissances du corps, Jahan esquisse l'histoire de la métaphore corporelle, qui est monnaie courante dans la littérature chrétienne et dans la relation de voyage missionnaire : «Hérité des temps médiévaux, le thème du corps mystique représentait la société chrétienne comme un assemblage organique où le Christ est bien évidemment la tête, tandis que les différents groupes sociaux occupent une place déterminée par référence aux fonctions et aux hiérarchies corporelles ${ }^{1}$. Mouvement, mortalité et communauté se croisent dans la définition même du corps à partir de l'analogie entre ce terme et « le total d'une communauté $^{2} »$. Veines du corps-État, les missionnaires assurent la circulation des ressources littérales et métaphoriques: ils portent secours aux membres lointains et retransmettent les richesses de ces contrées au cœur du corps du roi. Au champ lexical sanguin s'ajoute la métaphore de l'œil pour souligner que le corps-État surveille aussi ses membres. Le missionnaire se présente comme une sorte de gond, liant ensemble le monde, l'État et l'Église. À en croire Jahan, le corps sert de mesure et structure l'organisation du monde et de la société ; il offre une forme à l'abstraction de l'organisation civile, qui se reflète dans l'ordre plus grand de l'univers ${ }^{3}$. Et c'est précisément ce qui se produit dans la relation de Du Tertre.

Il faudrait dès lors se poser la question, à savoir comment est constitué ce corps communautaire colonial. D'abord, on peut noter qu'à la conceptualisation symbolique du corps s'ajoute une dimension concrète, physique : les habitants s'écroulent sous le poids du travail laborieux et s'affaiblissent à la suite

1 Sébastien Jahan, Les Renaissances du corps, p. 15.

2 Nous nous référons ici au Thresor de la langue françoyse, tant ancienne que moderne, de Jean Nicot (16o6), http://portail.atilf.fr/dictionnaires/TLF-NICOT/index.htm.

3 Sébastien Jahan, Les Renaissances du corps, p. 15. 
de maladies. Dès leur arrivée aux Îles, Du Tertre et les autres missionnaires doivent soulager une colonie entière qui se trouve dans la misère :

[Nicolas de la Mare] nous receut comme des Anges descendus du Ciel ; \& apres nous avoir mené dans nostre Chapelle de Nostre-Dame du Rosaire, \& qu'on eut chanté le Te Deum en action de grace de nostre heureuse arrivée, il envoya chercher de la Cassave pour nous donner à manger, n'en ayant pas un morceau dans sa case ; nous fusmes tous plus consolés de cette pauvreté, que si nous eussions trouvé toutes les mines d'or des Indes, chacun de nous s'estimant heureux de souffrir quelque chose pour la gloire de Iesus-Christ, en secourant ses membres. (1667, tome I :152)

Les expressions imagées situent l'arrivée à l'intérieur de la conception officielle et hiérarchique de la mission : Du Tertre et ses compagnons sont reçus « comme des anges descendus du Ciel ». Sont ensuite insérés dans le discours religieux des topoï antithétiques relatifs au Nouveau Monde : face à l'or des Indes est posée la pauvreté de la cassave. La mention de l'or fait bien sûr de nouveau allusion à la rapacité des Espagnols, mais sert surtout à illustrer l'idée que la richesse ne vaut rien en regard de la générosité d'une communauté d'amis. Du Tertre transforme ainsi savamment la pauvreté en argument propagandiste. L'image de la communauté misérable mais égalitaire, de concert avec l'évocation de la plante tropicale, devient le signe même de la promesse d'une communauté coloniale à venir comme partie constituante du corps du Christ. Inscrite dans une telle scène touchante, la motivation coloniale sous-entendue dans ce passage n'est pas de s'enrichir, mais de contribuer au bien-être des membres de l'Église.

La tranquillité de cet accueil modeste est aussitôt interrompue par une visite aux malades :

Cependant c'estoit la chose la plus pitoyable du monde à voir. Il y avoit presque 10o. malades au logis de M. de la Vernade, tous couchez sur la terre, ou au plus sur des roseaux, dont plusieurs estoient reduits aux abois, veautrez dans leurs ordures, \& sans aucun secours de personne. Ie n'avois pas plûtost fait à l'un qu'il falloit courir à l'autre. Quelques fois pendant que j'en ensevelissios un dans des feüilles de Bananier, (il ne falloit pas parler de toile en ce temps-là) je n'entendois par toute la case que des voix mourantes qui disoient, Mon Pere, attendez un moment, ne bouchez pas la fosse, vous n'aurez pas plus de peine pour deux ou pour trois que pour un seul : \& le plus souvent il arrivoit ainsi, car j'en 
enterrois assez communément deux ou trois dans une mesme fosse. (1667, tome I : 152-153)

Le passage est clairement marqué par la théâtralisation : il commence sur le mode de l'hyperbole, se focalise ensuite sur les corps malades avant de situer le sujet-narrateur au centre de la scène et de continuer sur le mode descriptif, où sont alors insérées les voix des mourants. Devant nous s'étale la scène larmoyante d'une foule de malades, abandonnés, déshumanisés, et au milieu desquels intervient le missionnaire. La maladie leur a ôté toute force; il ne leur reste que les mots pour exprimer de la pitié envers leurs confrères et l'espoir de recevoir l'extrême-onction. Les membres de la colonie ne sont que des corps, réduits à l'état d'automates en raison de la maladie. Dans ce monde de désespoir, le missionnaire effectue de son mieux son travail pour soulager les esprits. La pauvreté qui touche aussi les matériaux à disposition (feuilles de bananier au lieu de toiles) renforce l'image d'une colonie primitive obligée de recourir aux ressources et au savoir locaux. Du Tertre anime lui-même le passage en accourant tantôt auprès de l'un des mourants, tantôt auprès d'un autre. Puis, des voix suppliantes interviennent, moins pour crier désespérément au secours que pour manifester leur reconnaissance à l'égard du missionnaire et de la communauté coloniale.

Désormais, la mise en scène des colons souffrants ne fait plus seulement horreur, mais vise le cœur: Du Tertre semble chercher à toucher son lecteur par l'effet d'un mélange de dégoût et de sentimentalité éloquente par le biais de la voix dramatique des mourants. Nous sommes ici face à un autre type de drame colonial : un spectacle touchant qui sert en premier lieu à montrer que les habitants méritent le soutien de la France. L'argumentation s'effectue sans faire appel à la raison ou à la promesse d'une prospérité économique. En revanche, le missionnaire entend persuader grâce au pathos dont sont empreintes les voix agonisantes. En d'autres mots, l'exotisme se conjugue à une fonction pathémique : il s'agit de convaincre par le truchement des passions et d'aller « outre la raison », les voix mourantes touchant directement le cœur du lecteur. Et on peut juger de l'efficacité de ce procédé par la reprise qu'en fait un autre missionnaire dominicain, Chevillard, dans sa propre relation ${ }^{4}$.

4 André Chevillard, Les Desseins de son Éminence de Richelieu pour l'Amérique, p. 49-50. 
La scène précédente n'est pas la seule où les habitants français se présentent comme des enfants sollicitant un père. On pense aussi à celle où ces derniers viennent de s'installer à Saint-Martin, alors qu'ils se croient abandonnés par leur gouverneur Esnambuc, le fondateur des Antilles françaises. La colonie se trouve perpétuellement sous la menace des Anglais et des Amérindiens, et les habitants n'ont pas encore appris à cultiver la terre. De cette situation aussi, Du Tertre tire un tableau larmoyant, représentant des enfants délaissés par leur père: désespérés, les habitants « eurent recours aux larmes et aux regrets, \& passerent toute la nuict dans une tristesse qui n'est pas concevable». (1667, tome I : 33) Plus loin, à propos de la mort d'Esnambuc, il cite les mots qu'utilise Virgile pour louer César : «Les habitants l'ont pleuré comme leur Père ... » (1667, tome I : 119-120) Contrairement aux jésuites qui font surtout allusion à César en tant qu'empereur guerrier ${ }^{5}$, Du Tertre utilise encore l'analogie du père de famille.

En effet, toute la première partie historique est dominée par la métaphore corporelle, qui saisit la structure de la communauté et la fait correspondre à celle d'une famille. Patriarcale et hiérarchique, elle offre un ordre aux événements chaotiques des premiers établissements tout en attachant le nouveau membre au corps primaire et original. Selon Christopher L. Miller, le modèle familial représente une structure de pouvoir symbolique - l'Europe étant le père, l'Afrique la mère et les sociétés coloniales leurs enfants - qui non seulement se présente comme naturelle et éternelle, mais, qui plus est, se concrétisera plus tard dans le commerce triangulaire ${ }^{6}$. La hiérarchie sur laquelle repose cette configuration transforme le trope du père en une mise en abîme, posant les différents gouverneurs ou missionnaires en tant que pères et les habitants en tant qu'enfants, mais en esquivant le rôle de la mère. Le modèle de la famille se répète ensuite et s'autoconfirme à l'infini.

À maintes reprises, Du Tertre s'attribue lui-même la place du père, ce qui n'est guère étonnant étant donné sa profession. Mais plus que cela, cette place renvoie à une position narrative qui lui permet de mieux construire une sorte de psychologie de l'établissement. Dans un passage où il raconte la mort d'un nombre considérable d'habitants de Saint-Christophe, on voit par exemple que Du Tertre ne se contente pas de causes externes. Au lieu d'interpréter cette

5 Marie-Christine Pioffet, Le Tentation de l'épopée, p. 24, p. 40-41 et p. 116.

6 Christopher L. Miller, The French Atlantic Triangle, p. 5. Voir aussi Doris Garraway, The Libertine Colony, p. 127-128, pour une analyse de la notion de famille dans un contexte colonial. 
tragédie comme résultant uniquement de la famine et de la maladie, il tente de la cerner aux termes d'une problématique d'ordre psychologique: «Ie pense qu'il y avoit un peu de l'un et de l'autre : surtout je crois que la tristesse, que les nouveaux venus de Saint Christophe eurent de se voir empeschez de faire leur profit, comme on leur avoit promis, en fit plus mourir que toute autre chose. » (1667, tome I : 152) Les membres de la famille coloniale - les habitants - sont présentés comme des enfants suppliants qui ont besoin de la protection et de la direction de leur père, mais ils ont aussi certaines exigences ; ils veulent profiter de nouvelles terres. En jargon moderne, il s'agirait d'une sorte de dépression à la suite de rêves non réalisés qui leur aurait ôté le goût de la vie. Pour Du Tertre, la santé physique est liée à la santé mentale, et ces enfants de la colonie sont déçus puisque leur père n'a pas tenu sa promesse d'une vie prospère. On pourrait traduire le profit de la colonie par l'idée du sang de ce corps français maintenant étendu aux Amériques. Sans le profit qui motive les habitants à faire circuler le sang, ce nouveau membre risque d'expier.

Il est ainsi significatif que Du Tertre configure les obstacles de la colonisation comme un drame familial plutôt qu'une guerre. La narration - depuis l'établissement précaire jusqu'à l'aboutissement d'une véritable colonie - repose sur cette structure. Les souffrances physiques, les scènes larmoyantes et pathétiques, les anecdotes spectaculaires relatives aux corps des autres forment bien le drame de la colonisation. Et à travers ce drame, Du Tertre ne cherche pas seulement à mettre en scène le spectacle des corps interagissant dans un ailleurs lointain ; son but est également d'en appeler aux sentiments du lecteur. La mise en abîme de la hiérarchie politique catholique française s'inscrit dès lors dans l'écriture sur un mode sentimental. Reposant sur un certain degré d'identification, les passions, provoquées d'abord par la pitié, contribuent à rapprocher les mondes, comme dans une sorte d'exotisme à rebours.

\section{La voix touchante de l'autre}

Qu'en est-il des Amérindiens et des Africains dans ce drame familial de l'histoire coloniale? Leurs destins peuvent-ils émouvoir le lecteur? On retrouve en effet une fonctionnalité analogue au cadre familial dans la description des Africains, bien qu'elle soit beaucoup moins élaborée que dans les passages relatifs à la colonie française. Du Tertre souligne de plusieurs manières à quel point les esclaves tiennent à la famille et distingue cette qualité comme un trait de caractère (plutôt que comme le résultat des circonstances extérieures et historiques) qu'il inscrit dans le registre de la pitié. Ils ne supportent pas que l'on punisse leurs enfants «\& ne témoignent un extreme déplaisir du mal 
qu'on leur fait, car ils aymeroient mieux qu'on leur frappast qu'eux, \& ie n'ay iamais veu les esclaves moins maistres de leur colere, que quand il s'agit de l'interest de leurs pauvres enfans ». (1667, tome II : 510) Il raconte en détail comment l'esclave de sa paroisse en Guadeloupe, Dominique, amène ses enfants pleurer devant la tombe de sa femme (1667, tome II : 509) et comment les esclaves aident d'autres esclaves qui souffrent sous le joug d'un méchant maître. (1667, tome I : $5^{28}$ ) La fidélité profonde dont témoignent les Africains les uns aux autres selon Du Tertre s'étend aussi aux Blancs, à condition qu'ils soient de bons maîtres. Dans des scènes larmoyantes, il décrit par example comment plusieurs esclaves seraient venus au secours des missionnaires lors de la grande famine de la Guadeloupe. (1667, tome I : 498)

Dans un autre passage, Du Tertre insère une anecdote morale relatant les aventures d'une jeune esclave qui refuse de se marier parce qu'elle ne veut pas mettre au monde un enfant destiné à l'esclavage. Son maître se moque d'elle et l'amène à l'église pour un mariage forcé, mais elle y échappe grâce à son éloquence :

[...] elle respondit avec une fermeté qui nous étonna; non mon Pere, je ne veux ny de celuy-là, ny même d'aucun autre : je me cotente d'estre miserable en ma personne, sans mettre des enfants au monde, qui seroient peut-estre plus malheureux que moy, \& dont les peines me seroient beaucoup plus sensibles, que les miennes propres. (1667, tome II : 505)

Dans ce passage, c'est la jeune esclave qui s'exprime sur le mode sentimental à travers sa voix pathétique, dynamisant le discours pour susciter l'émotion du lecteur ${ }^{7}$. La scène remplit de toute évidence une fonction exemplaire, et le discours direct de l'esclave est fictionnel. Mais le but est clair et fait écho à une certaine forme de sentimentalité : l'auteur cite la femme pour que le lecteur ait pitié d'elle. Loin d'intervenir comme marque linguistique de différence, sa parole est rendue dans un français impeccable et sert plutôt à diminuer la distance entre le monde étranger et le lecteur. Du Tertre renforce et étend ainsi le portrait de la famille coloniale patriarcale à l'intérieur de laquelle les gouverneurs tiennent le rôle de pères veillant sur leurs enfants français, africains et amérindiens ${ }^{8}$.

7 Marc Bonhomme, Pragmatique des figures du discours, Paris, Honoré Champion, 2005, p. 166-71.

8 Voir Édouard Glissant, Poétique de la Relation, Paris, Gallimard, 1991, p. 59-75. Cela marque le commencement de ce que Glissant catégorisera quatre siècles plus tard comme une obsession de la filiation dans la pensée occidentale, étroitement liée à la colonisation. 
Les Autochtones jouent un rôle particulier dans la saga familiale du paradis colonial. Étant originaires des Îles, ils luttent pour leur droit à la terre. À ce sujet, arrêtons-nous sur un événement central de l'établissement français qui a inspiré plusieurs relateurs à en rapporter les événements. Il s'agit d'une guerre en Martinique, guerre contre les Amérindiens et provoquée par la mauvaise gouvernance de monsieur de l'Olive à la suite de la disparition du gouverneur Du Plessis en 1638, donc à l'époque où les Français partageaient les Îles avec les Autochtones et deux ans avant l'arrivée de Du Tertre aux Îles. L'importance de l'épisode est bien cernée par André Chevillard qui, dans ses Desseins de son Éminence de Richelieu pour l'Amérique, adopte un ton grandiloquent en préparant le lecteur à un épisode dramatique lorsqu'il annonce qu'il faudrait « un Amphithéâtre beaucoup plus grand que celuy des Romains, pour vous faire voir au iour les pieces diverses qui se passerent dans la conduite du Gouvernement de de l'Olive ${ }^{9}[\ldots] »$. Dans la version de Chevillard, Du Plessis meurt de désespoir en voyant souffrir son peuple. Quand Du Tertre relate le même épisode, il lui donne un ton à la fois tragique et galant: le bon gouverneur Du Plessis, s'opposant aux mauvaises intentions de son compagnon de l'Olive, meurt d'un excès de mélancolie après le décès de sa femme et la décimation de son peuple.

Du Tertre transforme les personnages historiques en personnages littéraires qui semblent agir selon une logique narrative et descriptive plutôt que selon la causalité d'un récit historique. Au lieu d'informer simplement le lecteur que Du Plessis refuse de participer au projet de de l'Olive d'attaquer les Amérindiens afin de s'emparer de leurs réserves de nourriture, Du Tertre préfère citer les paroles attribuées à Du Plessis. Ce «brave gentilhomme» dit à son compatriote qu'il «n'y pouvoit consentir, \& qu'il aymoit mieux perir avec tout son monde, que de se sauver par une voie si injuste \& si contraire aux intentions de sa majesté, \& aux desseins des seigneurs de la Compagnie ». (1667, tome I : 82) Le discours indirect, inséré dans des phrases à structure anaphorique, a ici pour effet de remplir une rhétorique politique de sentimentalité héroïque : Du Plessis aurait préféré la mort et celle de ses proches plutôt que de trahir sa tâche première officielle qui n'est ici nullement de s'enrichir, mais d'évangéliser les infidèles. Déçu par la réponse de Du Plessis, de l'Olive se tourne vers Esnambuc. Celui-ci résiste aussi en disant qu'il «n'y avoit rien de plus contraire aux ordres du Roy, \& des Seigneurs de la Compagnie, qui ayant pour but principal la conversion de ces infideles, vouloient surtout qu'on entretint la paix avec eux ». (1667, tome I : 83) Les paroles de Du Plessis et d'Esnambuc sont censées

9 André Chevillard, Les Desseins de son Éminence de Richelieu pour l'Amérique, p. 31. Charles de Rochefort raconte aussi cet épisode : Histoire naturelle et morale des îles Antilles de l'Amérique, p. 281. 
convaincre tant par les sentiments que par la raison. La vivacité qui caractérise le récit détaillé de la partie historique de la relation dutertrienne sert d'abord à établir ce lien permettant de capter le réel en touchant le lecteur plutôt que de raconter ce qui s'est réellement passé.

De l'Olive et Du Plessis représentent chacun une des facettes de la gouvernance coloniale: l'un sert le bien de la communauté et facilite le travail de peuplement en cherchant l'accord avec les nations voisines (les Amérindiens et les Anglais), tandis que l'autre agit en tyran en favorisant l'enrichissement personnel rapide, le bien de l'individu plutôt que celui du collectif, et la quête vaine de la gloire. Il n'y a pas de doute : la cible de la critique de Du Tertre, c'est de l'Olive, et la raison derrière l'antipathie qu'il lui voue est à trouver dans la politique missionnaire: ce gouverneur s'est emparé des terres des Frères Prêcheurs ${ }^{10}$. Mais cela, le relateur ne le dit pas ouvertement. Dans la deuxième édition, il amplifie le portrait de de l'Olive en lui conférant une sombre psychologie. À l'occasion de la négociation d'un traité avec des marchands de Dieppe contre l'avis du gouverneur de Saint-Christophe, de Poincy, de l'Olive tombe malade et doit se rendre à lîle de Niève, où il est atteint d'une «melancholie si estrange, qu'elle passa jusqu'à la phrenesie. On luy voyoit rouler les yeux à la teste, grinçant les dents, \& tous ses membres changez de posture par des convulsions épouvantables ». (1667, tome I : 144) Avec un tel portrait, qui n'est d'ailleurs pas sans rappeler les descriptions des danses des Amérindiens et des Africains, le lecteur ne s'étonnera pas qu'il abuse de son pouvoir. Créant ainsi un personnage malveillant et inconstant, Du Tertre personnalise sous forme de drame l'abus du système colonial, qui n'en est qu'à ses débuts, et l'oppose à la bonne gouvernance d'autres personnages comme Du Plessis et d'Esnambuc.

Après la mort de Du Plessis, de l'Olive retourne à la Guadeloupe, prend le pouvoir et ne manque pas l'occasion de partir à la recherche des Amérindiens ${ }^{11}$. En apprenant son dessein, ceux-ci prennent la fuite. Et quand les Français arrivent au village, ils ne trouvent «qu'un bon vieillard nommé le Capitaine Yance, âgé de plus de six-vingt ans, avec trois de ses fils \& deux autres jeunes sauvages ». (1667, tome I : 85 ) Ladjectif ainsi que la mention du nom propre du capitaine amérindien indiquent l'orientation de la sympathie de l'auteur.

10 Voir à ce sujet «Le Père Raymond Breton et ses manuscrits», introduction à Breton, Relation de l'île de la Guadeloupe, p. 12.

11 Tous les missionnaires donnent cette version des événements. Mais dans un article intitulé Les Exploits et logement des Français dans l'île de la Guadeloupe, et publié dans la Gazette le 30 mai 1638, Théophraste Renaudot prétend au contraire que ce sont d'abord les Amérindiens qui auraient attaqué les Français. Voir Ouellet (dir.), La Colonisation des Antilles I, p. 142. 
Du Tertre continue son récit en jouant sur le registre visuel, dramatique et sentimental:

[Yance] estoit sur le poinct de s'embarquer ; mais comme il vit les François venir à luy, il leur cria plusieurs fois, France non point fasche, ne se pouvant mieux expliquer : on luy dit qu'il n'avoit qu'à venir avec ses enfans en toute asseurance, \& qu'il ne luy seroit fait aucun tort. Sur cette promesse il y vint aussi-tost. $(1667$, tome I : 85)

L'inclusion du discours direct en italique dans le «baragouin », langage étranger, mais autre que la langue autochtone et plus proche du français, contribue à augmenter la tension dramatique de l'épisode. Il se crée un point de correspondance avec le lecteur propre au registre sentimental et d'autant plus fort que Yance s'exprime en une forme de français, même si ce n'est que d'une façon simple et «corrompue», comme disaient les relateurs, et se présente clairement comme un ami. Le capitaine est rapidement transformé en enfant naï victime du complot des Français, ce qui le place dans la ligne de la famille coloniale, tout en présentant les soldats de de l'Olive comme des traîtres.

Les mots énoncés par Yance avec sincérité agissent comme repoussoir face aux actions de de l'Olive et de ses gens, qui se saisissent de lui et de son groupe afin de les forcer à leur révéler où se trouve le reste de la nation caraïbe. Quand Yance et sa compagnie arrivent vers lui, de l'Olive change de «face \& de discours. » S'ensuit un échange rapporté au discours indirect: « [de l'Olive] l'appella plusieurs fois traître, luy reprocha que luy \& tous ses Compatriotes avoient conspiré contre la Colonie, \& resolu d'égorger tous les François: ce pauvre vieillard luy fit entendre que cela n'estoit point, qu'il n'y avoit jamais pensé $[\ldots]$ ». $(1667$, tome I : 85$)$ Bien que le dialogue entre de l'Olive et Yance dynamise le passage, il y a une différence quant à la façon dont leurs points de vue sont transmis. Tandis que de l'Olive «l'appelle traître » et lui fait «reproche », Yance pour sa part «fait entendre », ce qui suggère que sa façon de communiquer se base autant sur des gestes que sur des mots. L'absence de langue propre est en quelque sorte compensée par l'adjectif «pauvre» et par la rapide focalisation interne («il n'y avait jamais pensé ») qui oriente la sympathie du côté de Yance, bien que celui-ci n’ait pas accès à la parole.

On ne saurait surestimer l'importance de cet épisode, d'autant plus que Du Tertre l'étoffe dans la deuxième édition de son livre en y ajoutant un vocabulaire visuel et pathétique. Il s'agit du passage à l'intérieur duquel de l'Olive ordonne à l'un des fils de Yance d'aller chercher les femmes de son peuple, et ce dernier, au lieu de suivre les ordres des Français, s'enfuit avec elles. Nous avons affaire à une histoire enchâssée, encadrée par un commentaire 
introductif, et vivement dramatisée par le biais d'insertions de discours direct et indirect. De l'Olive a ici pris tous les traits d'un tyran : il complote, change d'humeur, abuse de la confiance d'autrui et ment afin de réaliser ses desseins. Aussi, Du Tertre attribue-t-il les qualités barbares aux Français, tandis que les Autochtones sont humanisés et individualisés : le capitaine amérindien a un nom et devient, face au mauvais comportement des Français, «ce pauvre vieillard », épithète répétée tout au long du passage. Puis, lorsqu'il assiste au meurtre brutal de son fils, il est présenté comme un «père affligé », pour magnifier le pathétique du passage.

Ce qui suit cette trahison mérite d'être cité dans son intégralité et avec ses variantes, bien que le passage soit long. Les phrases soulignées ont été ajoutées dans la deuxième édition. Lorsque Du Tertre a beaucoup modifié une phrase, la première version est mise en italique, entre parenthèses :

[de l'Olive] fit lier le vieillard, \& le fit monter dans sa Chaloupe avec un de ses Fils, lequel on poignarda un moment apres aux yeux de ce pere affligé. Cela fait, ces assassins, les mains rougies de sang, s'acharnerent sur ce pauvre vieillard, qu'une cruauté si barbare avoit également saisi \& de crainte \& d'horreur ; \& apres luy avoir furieusement enfoncé cinq ou six coups d'épées \& de coûteaux dans l'estomach \& dans le ventre, ils le jetterent lié, la teste en bas dans la Mer (Cela fait, ils vinrent au pere, qui estoit demeuré tout saisi d'une si horrible cruauté, \& après lui avoir don- cinq ou six coups de cousteau, \& cinq coups d'espée au travers du corps; ils le jeterent tout lié dans la mer, la teste en bas): mais comme il estoit d'une forte constitution pour son âge, \& qu'il faisoit encor quelque effort pour se sauver, s'estant délié un bras par son agitation, il nagea vers la Chaloupe, implorant par ses larmes \& ses cris la misericorde de ces impitoyables; mais ces tygres au lieu de s'amolir, par cruauté horrible l'assomerent à coups d'aviron.

Ils lierent les deux autres Sauvages, plus morts que vifs, \& leur firent commandement de les conduire au lieu où les femmes avoient fait leur retraitte ; l'un des deux appellé Marivet, fils du Capitaine Baron, si connu dans les Isles par l'inclination qu'il a tousjours eu pour les François, jugeant bien qu'il ne seroit pas plus favorablement traité que les autres, qu'il avoit veu massacrer, prit l'occasion d'une falaise, d'une hauteur prodigieuse, de laquelle il se précipita en bas dans des haziers, \& dans des ronces, sans se rompre aucun membre. Quoy qu'il se fut déchiré tout le corps, il ne laissa pas de se rendre le mesme jour à cinq lieuës de là, où estoient les autres Sauvages avec les femmes \& les enfants; il les avertit de ce qui s'estoit 
passé, \& de la resolution furieuse des François, qui ne les cherchoient que pour les mettre à mort.

Ie ne puis oublier la douceur \& la bonté naturelle de ce jeune Sauvage, qui montre bien qu'ils ne le sont que de nom, \& que le déreglement de la cholere rendoit nos gens plus sauvages \& plus barbares qu'eux. Ayant rencontré au milieu de tous ces Sauvages un garçon François; il ne luy témoigna aucun ressentiment de l'outrage qu'il avoit receu de ceux de sa nation ; \& au lieu de se venger sur luy, du sang qu'ils avoient si cruellement répandu, il se contenta de luy dire dans son baraguoin, ô Iacques, France mouche fâche, l'y matté Karaibes, c'est-à-dire, ô Iacques, les François sont extrémement fâchez, ils ont tué les Sauvages. (Remarquez icy un trait signalé de debonnaireté en ce Sauvage, qui contrecarre la cruauté \& barbarie des nostres. C'est qu'ayant rencontré au milieu de tous ces Sauvages un garçon François, sans luy tesmoigner aucun ressentiment, se contenta de lui dire dans son baragoin, ô Iacques, France mouche fasche, ly matté Karaïbes; c'est à dire Iacques les François sont extremément fâchez, ils ont tué les Sauvages.) (1654, tome I : 42-44; 1667 , tome I : 86-87)

La sympathie du narrateur est clairement orientée du côté de la victime, tandis que les compatriotes de Du Tertre sont métamorphosés en animaux qui ne s'expriment qu'à travers la brutalité de leurs actions ${ }^{12}$. On voit que le modèle du corps familial se transpose sur la représentation de l'autre, tandis que les Français sont des «assassins, les mains rougies de sang ». «Le barbare», écrit Requemora-Gros, « est celui qui ne comprend pas cette nouvelle esthétique et est étranger à cette forme d'ethos français ${ }^{13}{ }$. Dans le passage cité, ce sont les Français qui jouent le rôle des barbares, qui manquent de sensibilité et sont incapables de pitié. Et le lecteur qui aurait compris la nouvelle esthétique basée sur l'éthique regarderait avec horreur ses compatriotes et se tournerait vers les Amérindiens, comme si leurs voix s'adressaient directement à lui. Ce n'est que dans le dernier paragraphe que Marivet, le fils du Capitaine Baron qui s'est miraculeusement sauvé des Français en se jetant d'un rocher dans la mer, s'exprime en baragouin et interrompt la narration pour se faire entendre.

12 Du Tertre emploie ici une stratégie opposée à celle qu'utilisent les jésuites dans la Nouvelle-France. Les relations des jésuites tendent plutôt à animaliser les Amérindiens. Voir Marie-Christine Pioffet, La Tentation de l'épopée, p. 84. Charles de Rochefort aussi tend à animaliser les Autochtones et les appelle, entre autres, « tygres »: Histoire naturelle et morale des îles Antilles de l'Amérique, p. 424. 
Lapparition de « ce jeune Sauvage », descendant du Capitaine Baron, connu pour son amitié avec Raymond Breton et figure mythique dans la littérature des Îles, est intéressante. Il s'agit d'un ajout à la deuxième édition. Curieusement, on le retrouve cependant dans le manuscrit de 1648 , ce qui veut dire que la rencontre entre Du Tertre et le fils de Baron eut lieu avant la première édition en 1654. Il faut probablement mettre l'omission sur le compte de la rédaction rapide de cette version de l'ouvrage. Quoi qu'il en soit, l'inclusion de Marivet a pour résultat de consolider la dramaturgie de l'anecdote et de la rendre plus cohérente. L'échange entre l'Amérindien et le missionnaire clôt celle-ci et renforce sa portée émotionnelle par les paroles énoncées et la mémoire évoquée. De surcroît, cette tournure finale fait passer l'anecdote d'une énonciation narrative historique (par l'entremise du relateur-compilateur) à une énonciation discursive à la première personne mettant en scène Du Tertre lui-même comme témoin oculaire au deuxième degré. Ce changement constitue davantage une précision d'ordre rhétorique qu'une correction factuelle. Du Tertre nétait en effet pas encore arrivé aux Îles au moment des faits, mais il nous fait comprendre à travers le discours direct à quel point un des survivants du drame, Marivet, le fils de Baron, l'a marqué : il ne peut oublier la bonté naturelle dont témoignaient ses paroles.

Tout comme Du Tertre est touché par l'échange avec l'un des survivants du conflit, le lecteur devrait être affecté par le récit. Aussi passe-t-on d'un style plutôt commentateur à une description vive, exemple de cette figure de style que l'on appelle l'energeia, plaçant le drame directement sous les yeux du lecteur. Le passage inspire l'imagination et va droit au cœur au lieu de convaincre par un raisonnement logique ou moral, donnant au lecteur l'impression d'assister à la scène. Ruth Webb souligne cette capacité particulière de l'energeia, figure qui semble baliser le chemin qui mènera à l'avènement de l'esthétique et du sensible. L'effet de la figure de style n'est pas sentimental en soi, dit Webb, mais en affectant physiquement le lecteur, elle fait en sorte que ce dernier traverse un pathos similaire aux personnages impliqués dans les événements décrits ${ }^{14}$.

La présence de ces stratégies rhétoriques souligne à quel point l'écriture dutertrienne est orientée vers l'impact sur le destinataire. L'univers étranger n'est pas seulement intéressant comme objet à décrire; l'expérience de l'ailleurs est tout aussi propice, pour ne pas dire davantage propice, à captiver le

14 Ruth Webb, Ekphrasis, Imagination and Persuasion in Ancient Rhetorical Theory and Practice, London, Routledge, 2016, p. 100. «Even when the effect is not what we might strictly speaking call "emotional", the physical understanding of the impact of energeia means that a reader/listener who conceives an image of any kind in his or her mind is still undergoing a pathos of some kind as he or she experiences the word's effect. » 
public que les curiosités exotiques. Les stratégies d'écriture à base répétitive, qui dominent les illustrations et les passages introductifs, ne semblent donc pas suffire pour dire l'étranger. Bien au contraire, il faut combiner ces scènes douces avec des scènes macabres pour s'assurer de produire un effet de l'étranger sur le lecteur. Consciemment ou non, Du Tertre semble en effet devancer le goût de son temps. Car, comme l'a si bien démontré Requemora-Gros, «plus on approche du siècle de la sensibilité et plus l'esthétique de la pitié, proche du goût larmoyant, est développée ${ }^{15} »$. Au lieu d'évoquer directement les richesses ou bien de faire appel à la gloire impérialiste, son écriture se rapproche du mélodrame avant la lettre, anticipant un Diderot. Dans un sens, on pourrait dire que, s'il y a un Autre dans cette relation de voyage, c'est bien son destinataire et le relateur veut à tout prix le séduire.

Cet effet n'est pas sans implications symboliques. Si le lecteur est touché par le destin de l'étranger, cela veut également dire, par extension, que l'autre peut aussi trouver sa place dans la grande famille coloniale, telle une première articulation de l'assimilation, qui deviendra le trait caractéristique de la politique coloniale française. L'exotisme fait impression; il parle à l'imagination tout en proposant de penser une communauté coloniale. Il marque à la fois une fusion et une séparation d'avec des corps étrangers, exposant sa fonction symbolique aussi bien que stylistique et politique. Certes, la simplicité du langage et des mœurs autochtones dans la représentation qu'en fait Du Tertre placera l'étranger à un niveau inférieur de la hiérarchie globale du récit et de la société coloniale en général, mais son honnêteté correspond bien à l'éthique française et le rend digne d'appartenir à cette famille. Évidemment, il s'agit là d'une chimère. En réalité, on ne le sait que trop bien, l'actualisation de la nouvelle communauté mène à la disparition des Amérindiens et se construit sur le dos des Africains, qui ne seront pas, eux, inclus dans la famille du paradis colonial.

15 Sylvie Requemora-Gros, Voguer vers la modernité, p. 179. 